\title{
Simulation of single phase photovoltaic system based on fuzzy logic control with multilevel inverter
}

\author{
Ezzaldden Raweh ${ }^{1}$, Wei $\mathrm{Pi}^{1^{*}}$, Omar Busati ${ }^{3}$, Abdul Rehman ${ }^{2}$, Saif Mubbarak ${ }^{1}$, and Yinshun Wang ${ }^{2}$ \\ ${ }^{1}$ Key Lab. of HV and EMC Beijing, Electrical \& Electronic Engineering School, North China Electric Power University, Beijing 102206, \\ China. \\ ${ }^{2}$ State Key Laboratory of Alternate Electrical Power System with Renewable Energy Sources, North China Electric Power University \\ Beijing, 102206, China. \\ ${ }^{3}$ Key Lab. of Control engineering, School of Control and Computer engineering, North China Electric Power University Beijing, 102206, \\ China.
}

\begin{abstract}
To control the solar power, reliability and stability are two main challenges. In addition, the total harmonic distortion (THD) must be within limits for optimal operation. In an inverter, the harmonics are produced during the conversion of DC power to AC power, which will affect the power electronic devices. Therefore, to overcome such challenges in high voltage and high power systems multilevel inverter (MLI) topology is more useful. Such type of inverters uses various DC voltage levels to generate the stepped AC at its output, approaching the sinusoidal shape. The cascaded H-bridge, capacitor-clamped, and diode-clamped are the most commonly used multilevel inverters topologies. For photovoltaic (PV) usage, cascaded $\mathrm{H}-$ bridge (CHB) MLI is more adaptive among the three topologies, where for each H-bridge unit; each PV model behaves as an isolated DC source. This paper specifically focused on the simulation of PV power as a source to the system and displayed the potential of a single-phase 11-level CHB inverter. For switching the IGBT devices, sinusoidal pulse width modulation (SPWM) is applied. Moreover, the fuzzy logic control (FLC) is introduced to improve the power quality. FLC reduce the THD via finding the appropriate set of IGBT switch signals. To show the improvement in the operation and reduction in the complex harmony signal effects of the CHB 11-level inverter, the proposed system is designed in Matlab/Simulink software. Finally, the results show that the dynamic behavior of the FLC is much better than the traditional proportional integral derivative (PID) controller.
\end{abstract}

\section{Introduction}

Sun radiation is one of the significant source of renewable energy, the cleanest and environmentally friendly. Its source is the light radiation and heat coming out of the sun. People have advanced knowledge to exploit the clean sources in order to generate the power. An increased human activity related to energy that lead to discovered suitable generation methods. This energy is used in different applications: heating, water purification, electricity generation, etc. The electrical generation based on the photovoltaic (PV) cells is popular way to exploit the clean energy by utilizing PV phenomena to produce electrical energy. Further, to finding architectural plans that use solar power. Contemporary solar technology is separated into the negative and active division. Negative solar power utilizes heat or sun radiation light instantly, as in the constructions prepared to supply natural light from the sun. Active solar energy comprises photovoltaic and solar thermal methods.

Due to decreased costs and prices, installing the solar power has increased about $20 \%$ to $25 \%$ annually in the last two decades. This reduction in cost has led to enhance the performance of solar cells, manufacturing tools, and economic aspects 1 .

The focus of researchers is on the use of high penetration on the power system, and thus design and control the appropriate inverter for PV applications. Pulse width modulation (PWM) with electronic circuits is mainly utilized in power conversion frameworks to accomplish closed-loop control. Nevertheless, even upgraded PWM methods still unable to generate ideal waveforms, which are entirely subject to semiconductor switching frequency. Moreover, deformed waveforms lead to produce harmonics, causing losses of energy. Additionally, high-frequency noise leads to influence load power as well as the related controller 2 .

Recently, multilevel inverters have more attractive to minimize harmonic because of their advantages compared to traditional three-level PWM inverters. Besides, they can provide enhanced output wavelength, lower total harmonic distortion (THD), lower electromagnetic interference, and smaller filter size. The three typical topologies of the multilevel inverter are as follow: Cascaded H-bridge inverter, Capacitor clamped (flying capacitors), and Diode clamped (neutral clamped).

\footnotetext{
* Corresponding author: ppiiwei@ncepu.edu.cn
} 
The cascaded multilevel inverter is straightforward when compared with others because it does not require any flying capacitor and clamping diode. The capacitorclamped, diode clamped inverter need only one DC power supply, whereas the $\mathrm{CHB}$ inverter requires a separate DC power supply for each H-bridge. Due to this property of H-bridge, CHB-MLI is exceptionally suitable for solar applications; solar cells can be assembled as many separate generators. In this way, they meet the requirements of CHB-MLI. (Table 1)

Table 1. List of Abbreviations.

\begin{tabular}{|ll|}
\hline PV & Photovoltaic \\
CHB & Cascaded H-Bridge \\
MLI & Multilevel Inverter \\
PWM & Pulse Width Modulation \\
SPWM & Sinusoidal Pulse Width \\
FLC & Modulation \\
THD & Fuzzy Logic Control \\
PID & Total Harmonic Distortion \\
E & Proportional Integral Derivative \\
CE & Error \\
\hline
\end{tabular}

Moreover, advantages such as significant reduction in the power induced by sun darkening and the elimination of $\mathrm{dc} / \mathrm{dc}$ booster (needed to regulate the voltage levels) are also achieved. Hence a significant increase in efficiency and reliability 3 .

Multilevel inverter performance is determined principally through modification techniques. There are a few recognized pulse width modulation methodologies for the cascaded multilevel inverter such as sinusoidal PWM, space vector PWM, multicarrier PWM, and selective harmonics elimination 4. In comparison with the conventional strategy, the proposed approach is subjected to another formation plan that adopts the SPWM method that decreases the THD.

\section{Modeling and design CHB-MLI for PV applications}

The single-phase multilevel CHB inverter is described in Fig. 1 It consists of K-number of DC generators (PV) and CHB integrated into single-phase MLI topology. To achieve the required output current and voltage, each DC generator contains arrays of PV cells linked in parallel and/or series fashion, whereas H-bridges are made up of four transistors with a driver circuit and an antiparallel diode. For the desired level, the K-number of H-bridges rely on the number of desired levels, i.e., $n=2 k+1$, and it should be selected by considering two main parameters, i.e., the available design elements and the PV fields 5.

The sinusoidal waveform depends on the number of levels, where increasing the number of the levels will make the waveform more sinusoidal. But, increasing the number of the levels will increase both, the cost and complexity of the system while reducing the conversion frequency as compared to two-level converters.

As MOSFETs (low voltage transistors) pass considerably higher conversion frequencies than the IGBTs (high power transistors), so MLI can perform at substantially higher frequencies as compared to two-level converters. This feature permits the use of smaller low pass filters.

Each H-bridge is run by a square wave having an appropriate duty cycle or pulse width modulation shape, generating a ladder shape with or without pulse width modulation 6. In the view of the analysis of 230 volts, single-phase system, H-bridge units are aligned in five specific clusters, forming an 11-level inverter. Hence, it can be weighed as a valid trade-off among performance, cost, and complexity.

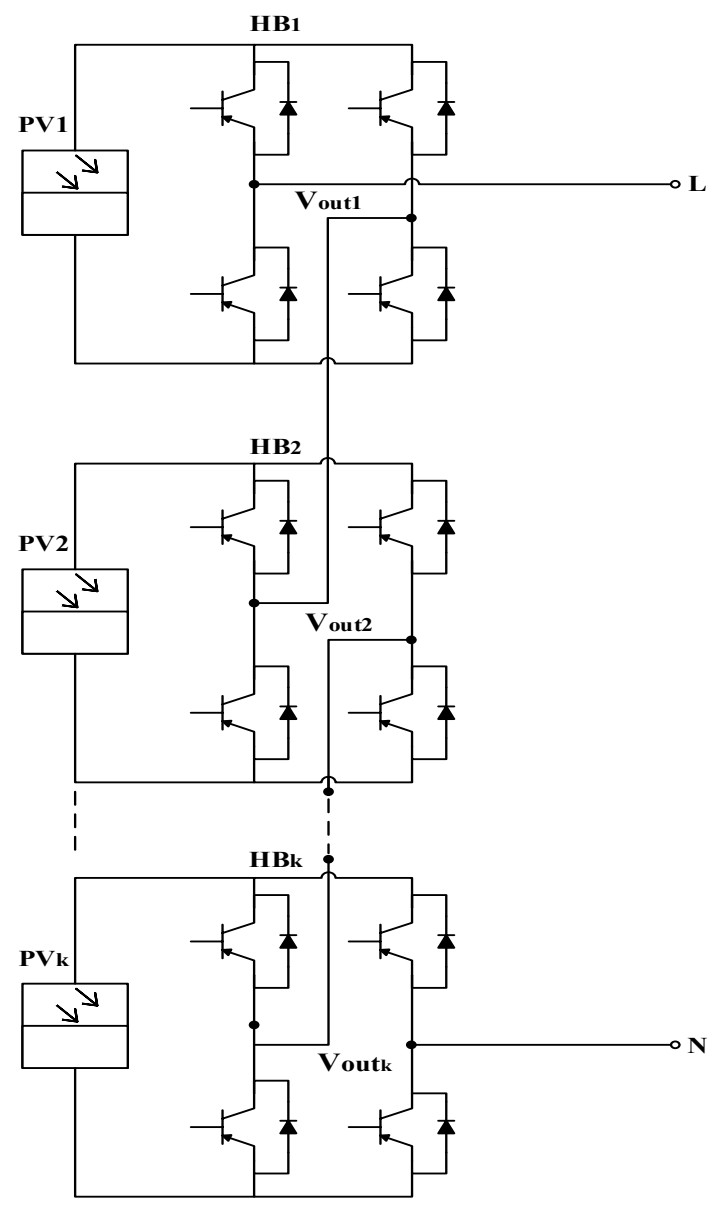

Fig. 1. Single-phase multilevel assembly of CHB inverter.

\section{Fuzzy Logic Controller with CHB-MLI and PV Array}

This segment demonstrates the capability of a SinglePhase CHB 11-level inverter represented by the FLC utilizing a PV power as a source.

Fig. 2 shows the general block diagram of the proposed control strategy. Which is consists of 11-level inverter; outcome signal of a PV system with a filter comparing with a reference voltage, the resultant signals are applied as an input to the fuzzy logic control. The output of FLC is processing with carrier modules by using a comparator to result in the switching signals. 


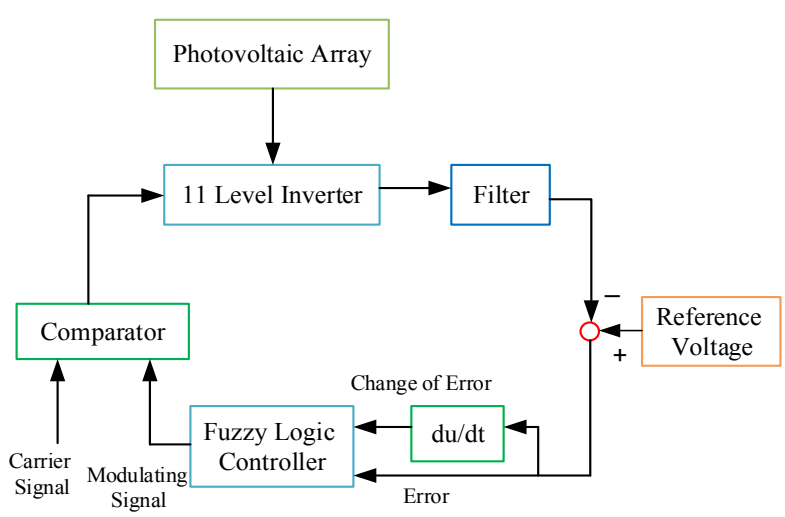

Fig. 2. The general block diagram of the proposed control strategy.

The fuzzy logic controller input factors are as per the following:

1. $V_{n}$ Filtered voltage output of the inverter $V_{\text {out }}$ divided by 100 ,

2. The difference in actual and reference signals is:

$$
A V_{\text {diff }}=V_{\text {out }} \text { inv }-V_{\text {ref }}
$$

After a low-pass filter, the $V_{o u t_{i n v}}$ measured at the terminals of the load. This option increases the quality of the control without presenting any retards; the bandwidth of the filter is selected about $1000 \mathrm{~Hz}$ with load resistive. The controller output is applied to gate drivers of an inverter. The output of FLC may presume eleven various states. The initial step amid FLC's design was to create a knowledge base (fuzzy rules), represented concerning statements, actions, and conditions. Beginning from the "TRUE" state (that is, the status is verified); a set of rules for errors was defined. Then, determine the conditions accordingly, and get a variable feedback. The crucial point of the controller is the type and number of membership functions (MFs). Its shape depends on the distribution of input data and can affect trace accuracy and execution time 7,8 .

The two input parameters of membership functions shown in Fig. 3(a) and (b). "NB," "NS," "ZE," "PS," and "PB" labels used for AVdiff are as per the following: "NB" = negative-big, "NS" = negative-small, "ZE" = zero, and so on.

It should be noted that, based on sensitivity analysis and simulations, to reduce output voltage variations to less than $10 \%, A V_{\text {diff }}$ is assumed to be within the range ($40,40) \mathrm{V}$.

The input and output variables for the fuzzy sets were nine, as the number of levels niv, niii, nii, ni, ze, pi, pii, piii, and piv. A Mamdani based system design has been achieved; Max-Min, configuration technique, and the center-of-gravity strategy were used in the inference engine and defuzzification procedure, respectively. The latter was embraced because it is a good trade-off between performance and complexity.

A large number of fuzzy ruling guarantee the interpolation as well as the applicable solution of the control device, and therefore, the high accuracy of control.

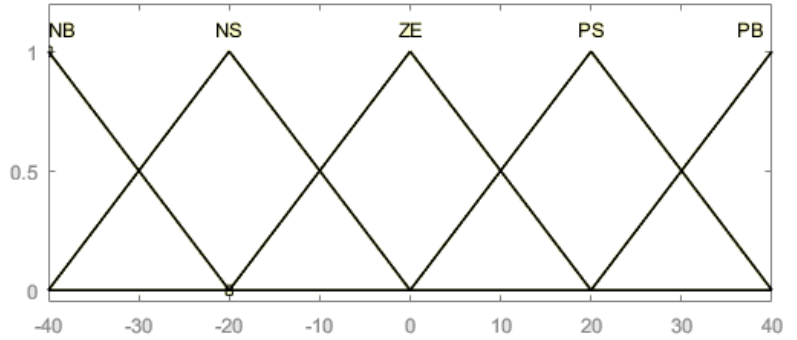

(a)

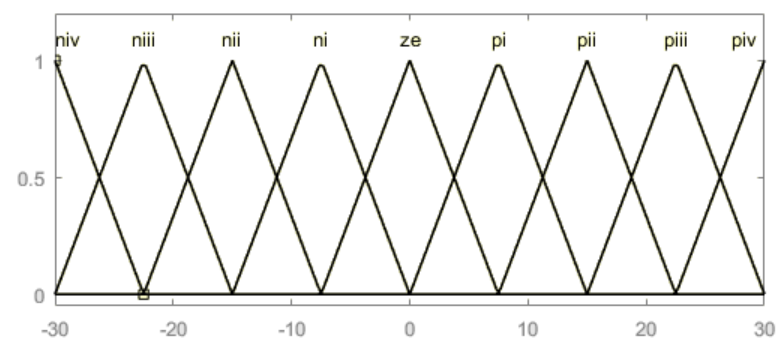

(b)

Fig. 3. (a) $A V_{\text {diff, }}$ (b) $V_{n}$ the input parameters of membership functions.

Since both quantity and type affect the fuzzy conjecture error, a large number of the ruling can cause an over parameterized system. As a result, decreasing the accuracy, generalization ability, and expanding the time of operation. The fuzzy rules rely on the execution time, selected membership functions, system performance, and the number of variables entered, adaptability and the ease of construction. In this article, the number of control rules and type are determined by sensibility study, which is done by changing the type and number of rules.

After a tuning process, sufficient level of performance was achieved, i.e., initiating from primary heuristic rules and reforming their type and number progressively.

Table 2. Rules of Inference.

\begin{tabular}{|l|lllll|}
\hline \multirow{2}{*}{\multicolumn{1}{|c|}{$\mathbf{V}_{\boldsymbol{n}}$}} & \multicolumn{5}{|c|}{$\mathbf{A V}_{\text {diff }}$} \\
\cline { 2 - 6 } niv & NB & NS & ZE & PS & PB \\
niii & niii & niii & niv & niv & niv \\
nii & nii & nii & niii & niv & niv \\
ni & ni & ni & nii & niii & niii \\
ze & ze & ze & ni & nii & nii \\
pi & pi & pi & ze & ni & ni \\
pii & pii & pii & pi & ze & ze \\
piii & piii & piii & pii & pi & pi \\
piv & piv & piv & piii & pii & pii \\
& piv & piv & piv & piii & piii \\
\hline
\end{tabular}

After the completion of this process, the 45 rules of inference shown in Fig. 4 and compiled in Table 2 were chosen. In Fig. 4, the X-axis shows the values for $A V_{\text {diff, }}$, the $\mathrm{y}$-axis the values for $V_{n}$, and the z-axis describes the state ahead assessed by FLC. For creating the rules of inference, the following logic was selected:

1. The inverter keeps its current state if the current state is correct, i.e.

$$
A V_{\text {diff }}=Z E
$$


2. Regarding a general state, the inverter state should

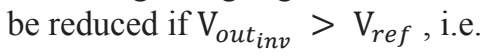

$$
A V_{\text {diff }}>0
$$

3. Similarly, the inverter state should be increased if

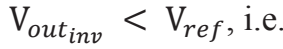

$$
A V_{\text {diff }}<0
$$

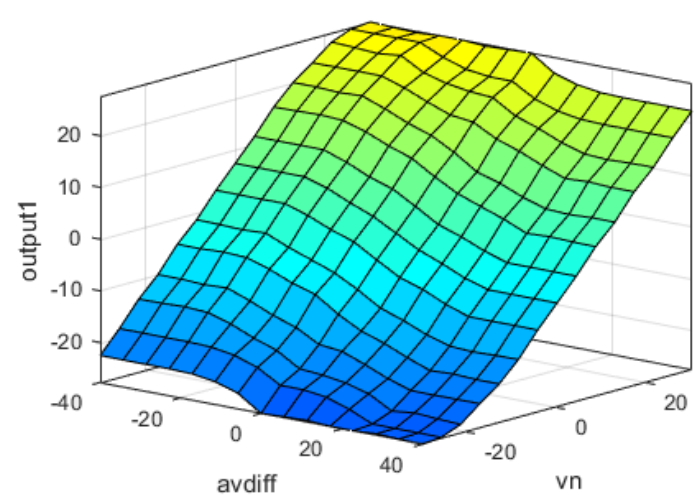

Fig. 4. 3D conception rules of inference.

\section{SPWM method}

In inverters, to reduce low order harmonics, PWM technique is widely used. During each half cycle, the PWM inverter switching frequency under regulating is highly amount, and by changing the pulse width, the output voltage is controlled 9. For each period, the SPWM systems are described by pulses with constant amplitude and, variable duty cycle. To decrease the inverter harmonic content and to gain the output voltage control, the width of those pulses is modified. SPWM strategy is generally applied in the applications of motor and inverter control 10 . To check the capability of the suggested MLI methodology in combining the output voltage with the desired amplitude and to get better harmonic spectrum, SPWM strategy is adopted to decide the recommended switching angles. To remove the ' $n$ ' harmonics, and to control the primary output voltage ' $n+1$ ' equations are required. Admirably, the switching angles need to be determined for the primary voltage V1, so that the output voltage

$$
V_{O}(\omega t)=V_{1} \sin (\omega t)
$$

And the particular higher harmonies for

$$
V_{n}(n \omega t)=0
$$

The third order harmonics are eliminated in a balanced three-phase system according to three-phase theory.

An iterative technique such as Newton-Raphson approach can be used to solve the set of nonlinear transcendental equations 11 . To solve the polynomial equations, the theory of resultants has been used which produces all possible solutions in contrast to repetitive methods 12. As the harmonic content characterized by transcendental equations, so for easiness they can be changed into polynomial equations. At this point, the resulting technique is utilized to get solutions when they existed. Finally, these sets of solutions must be analyzed for its THD to choose the set, which creates the minimal harmonic distortion.

\section{Simulation results}

In this paper, the simulation results are achieved using Matlab/Simulink.

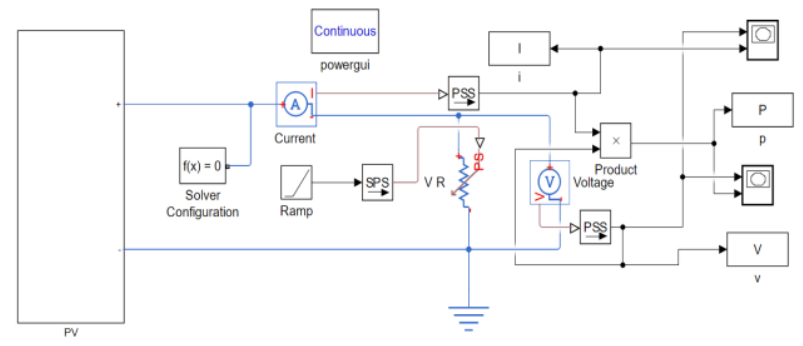

Fig. 5. Simulink model of PV panels

The PV panels model shown in Fig. 5. Output voltage is obtained as $46.64 \mathrm{~V}$ at maximum operation voltage, as seen in Fig. 6(a).

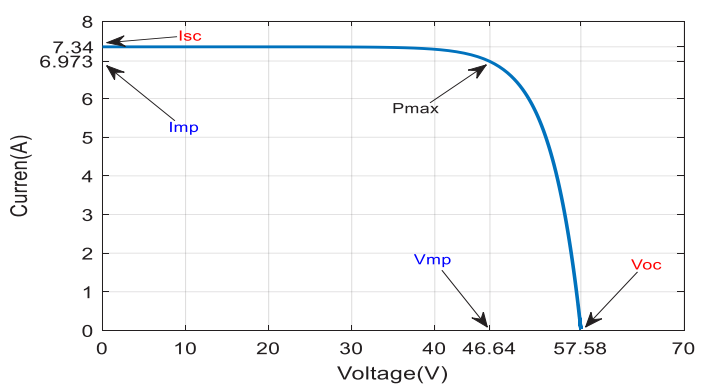

(a)

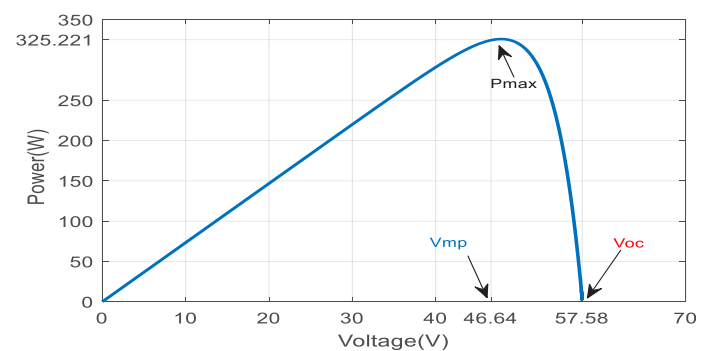

(b)

Fig. 6. (a) I-V curve, (b) P-V curve of PV panels

The total maximum power gives $325.221 \mathrm{~W}$ as result of multiple array current and voltage, as shown in Fig. 6(b). The PV model parameters are shown in Table 3.

Table 3. Solar Panels Parameters.

\begin{tabular}{|ll|}
\hline Parameters & Values \\
\hline Number of series cells & 96 \\
Maximum power, $\mathrm{P}_{\max }$ & $325.221 \mathrm{~W}$ \\
Maximum power voltage, & $46.64 \mathrm{~V}$ \\
$\mathrm{~V}_{m p}$ & \\
Maximum power current, & $6.973 \mathrm{~A}$
\end{tabular}




\begin{tabular}{|ll}
$\mathrm{I}_{m p}$ & \\
Open circuit voltage, $\mathrm{V}_{o c}$ & $57.58 \mathrm{~V}$ \\
Short circuit current, $\mathrm{I}_{s c}$ & $7.34 \mathrm{~A}$ \\
Temperature & $25^{\circ} \mathrm{C}$ \\
Solar irradiance & $1000 \mathrm{~W} / \mathrm{m}^{2}$
\end{tabular}

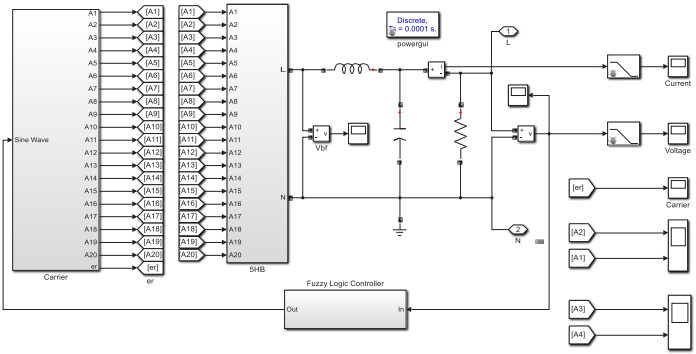

Fig. 7. Simulink model of the 11-level inverter with the fuzzy logic control circuit.

Fig. 7 show the closed loop formation of the 11-level inverter with fuzzy logic control. The filtered output voltage is achieved from 11-level inverter through a lowpass LC filter. The measured voltage feedback signal is compared with the reference signal as an error voltage, which is applied as an input to the FLC. There are two input signals an error (E) and change of error (CE), which feed the FLC unit. With the help of preceding error voltage signal's sample value, the change of error can be achieved. The task of the fuzzy logic controller is to do fuzzification, to convert the applied crisp inputs, then manipulating fuzzy set in accordance to fuzzy rules and membership function as evaluation mechanism, which is called the fuzzy inference system. The fuzzy sets which are the output of the inference system, re-converted by the defuzzification technique into the crisp output. This modulator output is matched with a triangle wave to provide pulses for 11-level inverter switches.

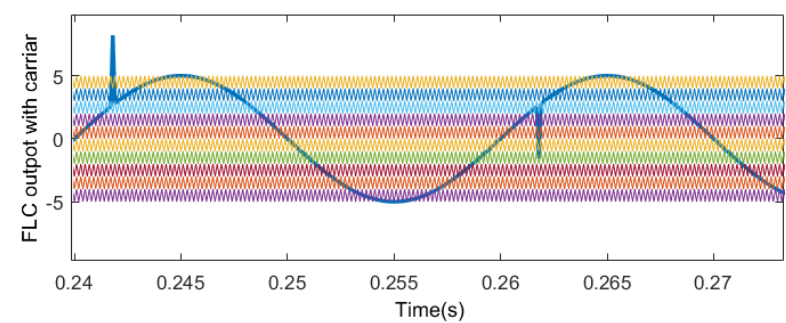

Fig. 8. The FLC output with the carrier signals

Fig. 8 represented the outcome of FLC with the carrier signals to produce 20th switch signals to trigger the H-bridge. Fig. 9 shows the two opposing converter switch signals.
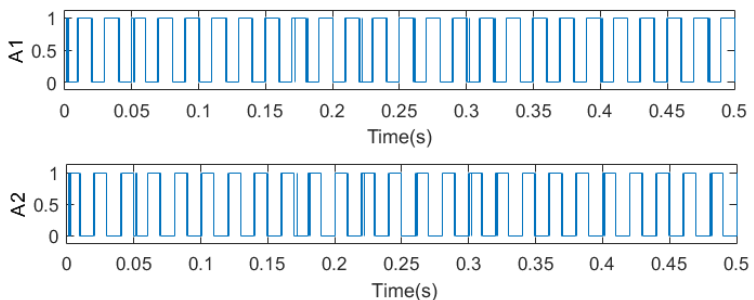

Fig. 9. The two opposing converter switch signals.

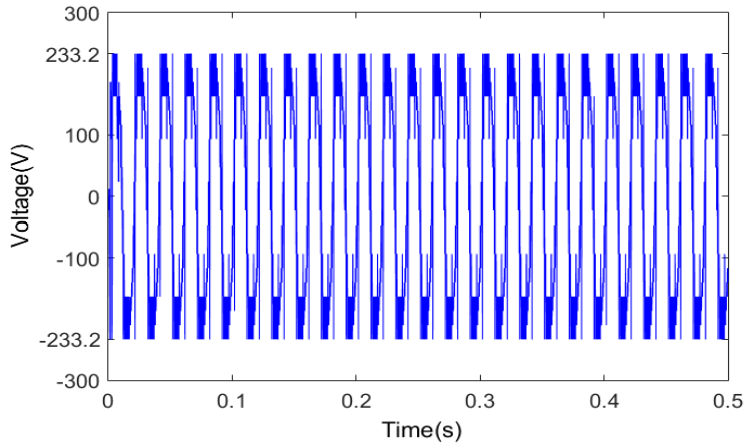

(a)

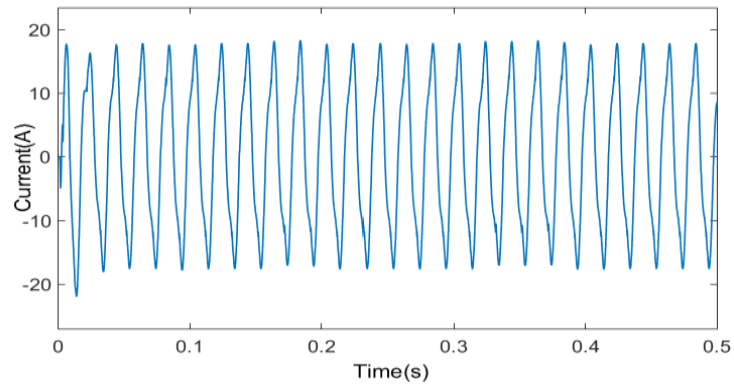

(b)

Fig. 10. (a) Voltage waveform, (b) Current waveform before the filter.

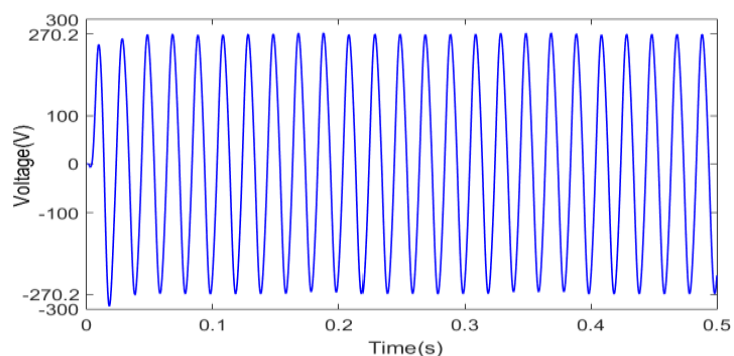

(a)

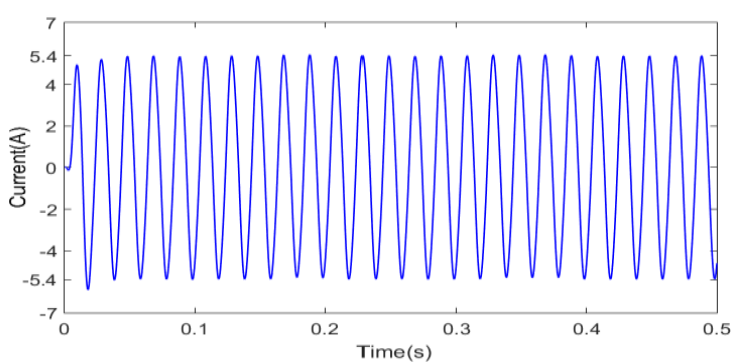

(b)

Fig. 11. (a) Voltage waveform, (b) Current waveform after the filter. 
The 11-level unfiltered output voltage and current is shown in Fig. 10(a) and (b). After passing through the LC low-pass filter, the output voltage and current are shown in Fig. 11(a) and (b) respectively. The THD is calculated using FFT to a measured voltage with considering 20 cycles and frequency $50 \mathrm{~Hz}$ to obtained the low percentage of total harmonic distortion about $3.62 \%$ as shown in Fig. 12.

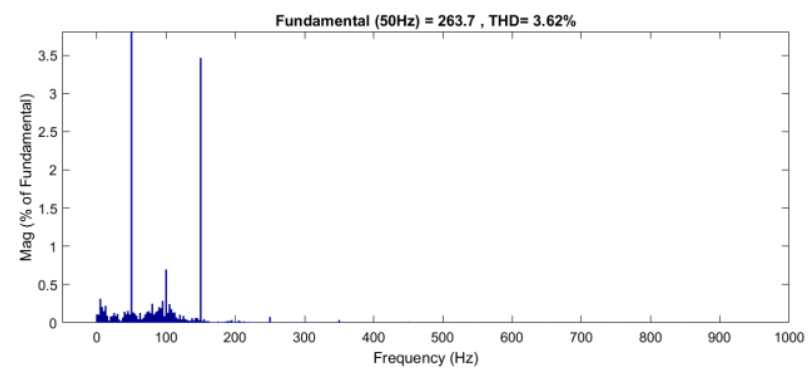

Fig. 12. FFT analysis for 11-level inverter output voltage after the filter.

The multilevel inverter parameters are presented in Table 4.

Table 4. Parameters of CHB Inverter.

\begin{tabular}{|ll|}
\hline Parameters & Values \\
\hline Number of H-bridge & 5 \\
Number of switches & 20 \\
Operation solar voltage & $46.64 \mathrm{~V}$ \\
Fundamental frequency & $50 \mathrm{~Hz}$ \\
Load resistor & $50 \Omega$ \\
Inductor & $20 \mathrm{e}-3 \mathrm{H}$ \\
Capacitor & $150 \mathrm{e}-6 \mathrm{~F}$ \\
\hline
\end{tabular}

\section{Conclusion}

The single-phase PV system using the CHB 11-level inverter based on fuzzy logic control has been introduced in this paper. The FLC Mamdani type with the 45 rules and the ways of Implication, Aggregation and Defuzzification have been specified as min, max and centroid respectively to produce the appropriate modulation signal. Which interaction with The SPWMshift phase carrier technique for firing the IGBT gates by pulse switch signals. Further, the proposed control method for adjusting the offset of modulation signal depend on the controlled voltage deviation and change of deviation with increase the stage of $\mathrm{CHB}$ to 11 levels is able to Keep the PV system successfully operation within acceptable inverter harmonic distortion. Additionally, the CHB converted the DC voltage of $\mathrm{PV}$ to $\mathrm{AC}$ with arising the peak amount of voltage about $19 \%$. The dynamic performance of the proposed control shows satisfactory performance over the conventional two-level inverter which has more details in 13. The proposed controller manipulated and reduced the total harmonic distortion within the allowable range of operation.

The fuzzy logic control technique enhanced the performance of the solar model by selecting the appropriate switch signals that are applied to the H-bridge inverter than the PID controller, which is introduced in 14.

The proposed methods can be extended for three Phase grid-connected under grid harmonic distortion or stand-alone as future work.

This work was supported in part by the National Natural Science Foundation of China under Grant 51877083 and by the Fundamental Research Funds for the Central Universities under Grant 2018MS004.

\section{References}

1. J. Carrasco, L. Franquelo, J. Bialasiewicz, E. Galván, R. P. Guisado, M. Prats, J. León, N. M. Alfonso, "Power-Electronic Systems for the Grid Integration of Renewable Energy Sources: A Survey," IEEE Trans. Ind. Electron, vol. 53, no. 4, pp. 1002-1016, (2006)

2. J. Selvaraj, N. Rahim, "Multilevel Inverter for GridConnected PV System Employing Digital PI Controller," IEEE Trans. Ind. Electron, vol. 56, no. 1, pp. 149-158, (2009)

3. S. Khajehoddin, A. Bakhshai, P. Jain, "The application of the cascaded multilevel converters in grid-connected photovoltaic systems," IEEE Canada Electrical Power Conference, (2007)

4. L. M. Tolbert, S. Member, F. Z. Peng, "Multilevel converters for large electric drives," IEEE Trans. Ind. Appl, vol. 35, no. 1, pp. 36-44, (1999)

5. F. T. Josh, Jovitha Jerome, J. Arulwilson, "Fuzzy Logic based nine level Inverter for photovoltaic systems," Eur. J. Sci. Res, vol. 78, no. 3, pp. 522-533, (2012)

6. L. Franquelo, J. Rodriguez, J. Leon, S. Kouro, R. Portillo, M. Prats, "The age of multilevel converters arrives," IEEE Ind. Electron. Mag, vol. 2, no. 2, pp. 28-39, (2008)

7. A. Green J. Z. Sasiadek, "Heuristic design of a fuzzy controller for a flexible robot," IEEE Trans. Control Syst. Technol, vol. 14, no. 2, pp. 293-300, (2006)

8. J. M. Mendel, G. C. Mouzouris, "Designing fuzzy logic systems," IEEE Trans. Circuits Syst. II, Analog Digit. Signal Process, vol. 44, no. 11, pp. 885-895, (1997)

9. K. B. Bhaskar, T. S. Sivakumaran, M. Devi, "Analysis of asymmetrical cascaded multi-cell multilevel inverter employing multicarrier level shifting pwm technique on different loads," IDNJST, vol. 9, no. 31, (2016)

10. A. S. Kale, A. V. Tamhane, A. A. Kalage, "Comparative study of SPWM and SVPWM cascaded h-bridge multilevel inverter," in Proceedings of 2017 International Conference on Intelligent Computing and Control, I2C2 (2017, 2018)

11. S. K. Sahoo, T. Bhattacharya, "Phase-Shifted Carrier-Based Synchronized Sinusoidal PWM Techniques for a Cascaded H-Bridge Multilevel 
Inverter," IEEE Trans. Power Electron, vol. 33, no. 1, pp. 513-524, 11 (2018)

12. C. Cecati, F. Ciancetta, P. Siano, "A Multilevel Inverter for Photovoltaic Systems with Fuzzy Logic Control," IEEE Trans. Ind. Electron, vol. 57, no. 12, pp. 4115-4125, (2010)

13. A. B. Patel, V. J. Anghan, P. B. Anghan, C. M. Vora, "Two Level and Five Level Cascaded H-bridge Inverter Structure with Amplitude Modulation ( AM )
Technique with Reduction in Total Harmonic Distortion," Int J Eng Res Appl, vol. 5, no. 11, pp. 133-137, (2015)

14. M. S. Sivagamasundari, P. Melba Mary, "Real Time Implementation of PI and PID Controlled Cascaded H-Bridge Eleven Level Inverter using SPWM," INFORM MIDEM, vol. 46, no. 2, pp. 65-73, (2016) 